(c) Elsevier/INRA

Article original

\title{
Paramètres génétiques de croissance et de gavage de la cane commune
}

\author{
B Poujardieu ${ }^{1}$, F Guichard ${ }^{2}$, P Laventure ${ }^{2}$ \\ 1 Institut national de la recherche agronomique, \\ station d'amélioration génétique des animaux, \\ BP 27, F31326 Castanet-Tolosan cedex; \\ 2 Sica-Sepalm, route de Meilhan, F40250 Souprosse, France
}

(Reçu le 13 Janvier 1994; accepté le 20 avril 1994)

Résumé - Nous analysons les performances de 5595 mulards mâles, gavés en ferme en 39 lots, fils de 93 mâles de Barbarie de 3 souches, une par année (1990-1992), et de 1069 canes de la souche Aliénor d'origine de race Pékin sélectionnée à la Sica-Sepalm. Nous estimons par maximum de vraisemblance restreint les paramètres génétiques de la souche maternelle; le modèle prend en compte les effets fixés du lot de gavage et du mâle de Barbarie et l'effet aléatoire de la mère des mulards. Les généalogies remontent aux fondateurs. Les valeurs des héritabilités et de leurs écarts types sont les suivantes : poids à la mise en gavage à l'âge moyen de $89 \mathrm{j}: 0,33(0,01)$, poids du paletot (peau, gras, muscles et os du dos, des jambes et des ailes) : $0,29(0,02)$, poids du foie : $0,17(0,01)$, poids de l'exsudat lipidique à la stérilisation : $0,18(0,01)$, poids du magret droit (muscle pectoralis major, peau et gras de couverture) : $0,22(0,01)$, poids du muscle du magret droit : $0,29(0,02)$, poids de l'ensemble peau et gras sous-cutané du magret : $0,13(0,01)$. La corrélation génétique entre le poids du foie et le poids de l'exsudat lipidique à poids d'échantillon constant est de $0,89(0,01)$; les corrélations génétiques entre ces 2 caractères et les autres variables sont similaires : le poids du foie est, génétiquement aussi, le facteur déterminant de la fonte lipidique à la stérilisation. Il existe un antagonisme génétique entre le poids du foie et le poids à la mise en gavage $(-0,14(0,05))$, le poids du paletot $(-0,23$ $(0,07))$, le poids du magret $(-0,16(0,01))$ et de ses constituants, muscle $(-0,17(0,06))$ ou peau et gras $(-0,06(0,07))$. Les corrélations génétiques entre le poids à la mise en gavage, le poids du magret et de ses constituants sont comprises entre 0,57 $(0,05)$ et 0,91.

canard Pékin / héritabilité / corrélation génétique / croissance / gavage

Summary - Genetic parameters for growth and cramming traits in common ducks. Data on 5595 male mule ducklings, 93 Muscovy drakes and 1069 dams of the Aliénor strain derived from the Peking breed and selected by Sica-Sepalm Co recorded over 3 years (1990-1992) were analyzed. Dams were selected to improve prolificity, growth and cramming traits. The ducklings were force-fed in 39 cramming lots. The genetic parameters of the maternal strain were estimated by an animal model including a fixed effect of the 
cramming lots, a fixed effect of the Muscovy drake, and a random effect of the mother of the ducklings. A restricted maximum likelihood estimator was used. The pedigrees were traced to the founders. Heritabilities and their standard errors were: weight at $89 \mathrm{~d}$ old, beginning of the cramming period 0.33 (0.01); 'paletot' (skin and backfat, and skin fat, bone, and muscle of the legs and the wings); weight 0.29 (0.02); liver weight 0.17 (0.01); fat release at the sterilization 0.18 (0.01); right crammed breast muscle, skin and fat ('magret') weight 0.22 (0.01); right crammed breast muscle weight 0.29 (0.02); and skin and fat weight of the breast muscle $0.13(0.01)$. The genetic correlation between the liver weight and the fat release is 0.89 (0.01); the correlations between these traits and the others are similar; the liver weight is also the genetic trait determining the fat release at sterilization. An antagonism exists between the liver weight and the weight at $89 \mathrm{~d}$ old $(-0.14(0.05))$, the 'paletot' weight (-0.23 (0.07)), the weight of 'magret' $(-0.16(0.07))$ and its components, breast muscle (-0.17 (0.06)) and skin and sub-cutaneous fat $(-0.06(0.07))$. The genetic correlation values between the weight at $89 \mathrm{~d}$ old, the weight of the 'magret' and of its components falls between $0.57(0.05)$ and 0.91 .

Peking duck / heritability / genetic correlation / growth / force-feeding

\section{INTRODUCTION}

Le canard mulard, hybride intergénérique d'un canard mâle de Barbarie (Caïrina moschata) et d'une cane commune (Anas platyrhyncos), est l'un des animaux les plus utilisés pour la production de magret et de foie gras en France. Le mulard recherché par le sélectionneur est blanc; son poids est suffisant à la mise en gavage; il possède alors un filet développé; il donnera à la fin d'un gavage court et précoce un paletot et un magret aussi maigre que possible et un foie lourd de qualité; cet animal descend d'une mère prolifique. À moins de n'accepter qu'une progression minime sur chacun des caractères, il est impossible de les sélectionner tous en simultanéité. Des choix s'imposent. La connaissance des paramètres génétiques de la souche sélectionnée permet de mieux les raisonner.

Le but de cette étude est d'estimer les valeurs des héritabilités et des corrélations génétiques d'une population de cane commune sélectionnée à partir des performances de leurs fils gavés contrôlés en ferme.

\section{CONDUITE DES ANIMAUX ET CARACTĖRES ANALYSÉS}

Nous analysons les performances de 5595 mulards mâles, fils de 93 mâles de Barbarie de 3 souches, une par année (1990-1992), et de 1069 canes de la souche Aliénor d'origine de race Pékin sélectionnée à la Sica-Sepalm. Ces données sont collectées lors du testage en ferme sur descendance des canes de cette souche. Les canetons mulards naissent en 2 lots d'éclosion à $15 \mathrm{j}$ d'intervalle chaque année; les canetons d'un lot d'éclosion sont élevés ensemble; ils sont gavés en plusieurs lots de gavage, 39 au total.

Les conditions de milieu varient d'un lot d'élevage à un autre. Le plus souvent les canetons sont élevés en bâtiment sur litière ou sur caillebotis avec accès à un parcours. Jusqu'à l'âge de 6 sem ils reçoivent un aliment complet de démarrage 
fournissant en moyenne $2920 \mathrm{kcal} / \mathrm{kg}$ et $20,5 \%$ de MAT (matière azotée totale). De l'âge de 6 sem à l'entrée en gavage à l'âge de 12 sem ils reçoivent un aliment complet croissance-finition fournissant $2950 \mathrm{kcal} / \mathrm{kg}$ et $16,5 \%$ de MAT en hiver et $2800 \mathrm{kcal} / \mathrm{kg}$ et 15,5\% de MAT en été. Quinze jours avant le début du gavage, à l'âge de 10 sem, les canetons entrent en prégavage; ils passent d'une alimentation sinon ad libitum du moins libérale à une alimentation par repas à heure fixe dans la journée (Babillé, 1989). Les canetons sont gavés en 2 repas quotidiens sans repasse. Au moment de l'entrée en gavage l'âge et le poids des animaux sont enregistrés.

Les canetons sont sacrifiés en salles d'éviscération collectives. Après électronarcose, ils sont saignés, plumés puis éviscérés à chaud. Les foies sont placés en sacs plastiques sur un lit de glace pilée puis mis en chambre froide avec les paletots. Le lendemain les paletots sont pesés puis découpés et les magrets droits disséqués, le muscle Pectoralis major est séparé de l'ensemble de la peau et du gras sous-cutané. Le paletot est un ensemble comprenant la peau, le gras sous-cutané, les muscles et les os du dos, des ailes et de la cuisse. Le magret est l'ensemble constitué par le muscle Pectoralis major et la peau et le gras sous-cutané de couverture. La durée du gavage, le poids du paletot, le poids du foie, le poids du magret droit, le poids du muscle et le poids de l'ensemble peau et gras sous-cutané du magret droit sont les variables analysées.

Un échantillon de foie d'un poids d'environ $60 \mathrm{~g}$ est prélevé dans la partie médiane de la face dorsale du lobe droit pour étudier la qualité technologique. Elle est appréciée par le poids de l'exsudat lipidique à poids de l'échantillon constant; l'exsudat lipidique est la perte de poids de l'échantillon de foie soumis, en boîte sertie, à une stérilisation de valeur stérilisatrice égale à l'unité; dans notre cas, cette valeur est obtenue par un chauffage à $105^{\circ} \mathrm{C}$ pendant 55 min.

\section{MÉTHODE D'ANALYSE}

Les héritabilités et les corrélations génétiques entre les caractères sont estimées par la méthode du maximum de vraisemblance restreint (REML) appliqué à un modèle mixte selon la méthode et à l'aide du logiciel proposé par Groeneveld et Kovac $(1990 \mathrm{a}, \mathrm{b})$. Le modèle comporte 2 effets fixés, le mâle Barbarie père des mulards (93 niveaux) et le lot de gavage (39 niveaux) que l'on interprète comme l'association d'un effet temporel et d'un effet milieu particulier auxquels sont soumis les canetons gavés. L'effet aléatoire est celui de la valeur génétique additive de la cane commune mère des mulards. Les généalogies remontent jusqu'aux fondateurs. L'effet éventuel de la consanguinité sur les performances est négligé.

Les calculs sont réalisés sur l'ordinateur IBM 30.90 du centre de traitement de l'information du centre de recherche INRA de Jouy-en-Josas. Les fichiers sont préparés à l'aide de logiciels de la programmathèque SAS.

\section{RÉSULTATS}

Le tableau I présente les caractères zootechniques de l'échantillon. Les durées de gavage courtes, eg $8 \mathrm{j}$, sont celles de canetons éliminés du gavage pour des raisons diverses, inadaptation au gavage ou problème pathologique; ils représentent moins 
de $0,5 \%$ de l'effectif. Pour un gavage réalisé en moyenne en 30 ou 32 repas mais qui pour certains lots atteint 60 repas, la peau et le gras sous-cutané représente $37 \%$ du poids du magret.

Tableau I. Statistiques descriptives de l'échantillon de 5595 mulards mâles.

\begin{tabular}{lccc}
\hline Caractère & $\begin{array}{c}\text { Moyenne } \\
\text { (écart type) }\end{array}$ & Minimum & Maximum \\
\hline Âge mise en gavage (j) & $88,6(3,0)$ & 83 & 93 \\
Durée du gavage (j) & $15,5(2,6)$ & 8 & 30 \\
Poids début (g) & $3948(412)$ & 2390 & 6260 \\
Poids paletot (g) & $2392(265)$ & 408 & 3359 \\
Poids du foie (g) & $495(127)$ & 127 & 1001 \\
Poids du magret (g) & $369(44)$ & 218 & 548 \\
Muscle du magret (g) & $231(29)$ & 140 & 382 \\
Peau et gras magret (g) & $137(25)$ & 59 & 272 \\
Poids exsudat (g) & $18,9(9,0)$ & 1,7 & 72 \\
Taux de fonte (\%) & $31,9(15,3)$ & 5 & \\
\hline
\end{tabular}

Les estimations des héritabilités (tableau II) varient de 0,13 pour la somme des poids de la peau et du gras du magret à 0,33 pour le poids à la mise en gavage. Les estimations sont précises puisque les valeurs des écarts types n'excèdent pas 0,02.

Tableau II. Valeurs des estimées et des écarts types des héritabilités (sur la diagonale) et des corrélations génétiques (au-dessous) ou phénotypiques (au-dessus de la diagonale) : paramètres génétiques de la voie maternelle du croisement $\mho^{\prime}$ Barbarie $\times \bigcirc$ Pékin.

\begin{tabular}{lccccccc}
\hline & $\begin{array}{c}\text { Poids } \\
\text { début }\end{array}$ & $\begin{array}{c}\text { Poids } \\
\text { paletot }\end{array}$ & $\begin{array}{c}\text { Poids } \\
\text { foie }\end{array}$ & $\begin{array}{c}\text { Poids } \\
\text { exsudat }\end{array}$ & $\begin{array}{c}\text { Poids } \\
\text { magret }\end{array}$ & $\begin{array}{c}\text { Muscle } \\
\text { magret }\end{array}$ & $\begin{array}{c}\text { Gras } \\
\text { magret }\end{array}$ \\
\hline Poids début & 0,33 & 0,77 & $-0,03$ & $-0,12$ & 0,66 & 0,61 & 0,41 \\
Poids paletot & $(0,01)$ & & & & & & \\
& 0,89 & 0,29 & $-0,00$ & $-0,17$ & 0,78 & 0,66 & 0,58 \\
Poids du foie & $(\mathrm{nc})^{*}$ & $(0,02)$ & & & & & \\
& $-0,14$ & $-0,23$ & 0,17 & 0,75 & 0,03 & $-0,04$ & 0,09 \\
Poids exsudat & $(0,05)$ & $(0,07)$ & $(0,01)$ & & & & \\
& $-0,24$ & $-0,36$ & 0,89 & 0,18 & $-0,10$ & $-0,12$ & $-0,02$ \\
Poids du magret & $(0,05)$ & $(0,04)$ & $(0,01)$ & & & & \\
& 0,87 & 0,92 & $-0,16$ & $-0,26$ & 0,22 & 0,84 & 0,75 \\
Muscle du magret & $(0,01)$ & $(0,10)$ & $(0,01)$ & $(0,05)$ & $(0,01)$ & & \\
& 0,78 & 0,77 & $-0,17$ & $-0,25$ & 0,91 & 0,29 & 0,27 \\
Peau et gras & $(0,02)$ & $(0,01)$ & $(0,06)$ & $(0,05)$ & $(\mathrm{nc})^{*}$ & $(0,02)$ & 0,13 \\
du magret & 0,57 & 0,67 & $-0,06$ & $-0,15$ & 0,63 & 0,27 & 0,03 \\
& $(0,05)$ & $(0,03)$ & $(0,07)$ & $(0,06)$ & $(0,05)$ & $(0,06)$ & $(0,01)$ \\
\hline
\end{tabular}

* Écart type non calculé. 
Les valeurs des corrélations génétiques (tableau II) entre les caractères pondéraux, poids à la mise en gavage, poids du paletot, poids du magret et du muscle du magret sont fortes, de 0,57 à 0,91 ; elles sont supérieures aux valeurs des corrélations phénotypiques (tableau III).

Tableau III. Héritabilités estimées en race Pékin sur des animaux non gavés.

\begin{tabular}{|c|c|c|c|}
\hline Caractère & Sexe & Valeur & Auteur \\
\hline Poids $49 \mathrm{j}$ & $\mathrm{m}$ & 0,28 & Pingel et Jung (1979) ${ }^{a}$ \\
\hline Poids $50 \mathrm{j}$ & $m+f$ & 0,65 & Dujunov $(1965)^{\mathrm{a}}$ \\
\hline Poids $51 \mathrm{j}$ & $\mathrm{m}+\mathrm{f}$ & 0,76 & Clayton et Powell (1979) \\
\hline Poids $56 \mathrm{j}$ & $\mathrm{m}$ & 0,31 & Sochoka et Wezik $(1971)^{a}$ \\
\hline Poids $56 \mathrm{j}$ & f & 0,35 & Sochoka et Wezik $(1971)^{a}$ \\
\hline Poids $56 \mathrm{j}$ & f & 0,37 & Kosba et al (1981) \\
\hline Poids $56 \mathrm{j}$ & f & 0,32 & Kosba et al (1981) \\
\hline Poids $56 \mathrm{j}$ & $\mathrm{m}$ & 0,35 & Pingel et Heimpold $(1983)^{a}$ \\
\hline Poids $56 \mathrm{j}$ & $\mathrm{f}$ & 0,43 & Pingel et Heimpold (1983) ${ }^{\mathrm{a}}$ \\
\hline Poids $56 \mathrm{j}$ & $\mathrm{m}$ & 0,43 & Kain $(1988)^{a}$ \\
\hline Poids $56 \mathrm{j}$ & $\mathrm{f}$ & 0,41 & Kain $(1988)^{a}$ \\
\hline Poids éviscéré & $m+f$ & 0,74 & Clayton et Powell (1979) \\
\hline Muscle filet & $m+f$ & 0,51 & Clayton et Powell (1979) \\
\hline
\end{tabular}

${ }^{a}$ Résultats cités par Pingel (1990).

Le poids du foie et le poids de l'exsudat lipidique sont corrélés $(0,89)$; nous avons en effet considéré le poids de l'exsudat à poids d'échantillon constant et non à poids de foie constant, ce qui aurait été une bonne approximation du taux de fonte. Cependant puisque la valeur de cette corrélation n'est pas égale à l'unité, une variabilité génétique indépendante du poids du foie subsiste. Les valeurs des corrélations génétiques, non nulles, entre ces caractères et les autres variables sont semblables. Les corrélations génétique entre poids du foie, poids de l'exsudat lipidique et les autres caractères sont négatives. L'intensité de la liaison du poids $\mathrm{du}$ foie est plus forte avec le muscle du magret $(-0,17(0,06))$ qu'avec l'ensemble peau et gras sous-cutané du magret $(-0,06(0,07))$. Les précisions des estimations sont bonnes car les écarts types n'excèdent pas 0,07 .

Les valeurs des corrélations phénotypiques (tableau II) entre les poids du foie, de l'exsudat lipidique et les autres caractères sont faibles; elles ne sont négatives qu'avec la seconde variable. Au niveau phénotypique l'opposition entre l'exsudat et le poids du magret $(-0,10)$ est clairement due à une opposition d'état d'engraissement car la corrélation avec le muscle du magret est positive $(0,12)$. Les valeurs des corrélations phénotypiques ou entre les autres caractères, poids au début du gavage, poids du paletot, poids du magret et de ses constituants (muscle, peau et gras) sont fortes à moyennes (de 0,27 à 0,78 ). 


\section{DISCUSSION}

Les performances zootechniques moyennes de l'échantillon traité sont analogues à celles des populations de mulards testés par la Sica-Sepalm (Roussely et al, 1993); pour la qualité, nous considérons la totalité de l'information disponible. Les données antérieures à 1990 ne sont pas considérées car les pères étaient alors inconnus (Roussely et al, 1993). Dans cette étude la peau et le gras sous-cutané représente $37 \%$ du poids du magret. Pour un gavage en 36 repas Sazy et al (1993) trouvent une valeur de $39 \%$ et pour 28 repas de $36 \%$. Pour 24 repas Guy et al (1992) trouve $32 \%$ et Brun et al (1995) $42 \%$. Tous les mulards étudiés par ces auteurs sont des hybrides d'Aliénor. L'état d'engraissement des mulards, jugé par la proportion de peau et de gras sous-cutané dans le magret, semble donc moyen. Une situation de ce type correspond bien souvent à un gavage d'intensité moyenne; dans notre cas, le plus vraisemblable est une juxtaposition de lots de gavage de durées et d'intensités variables, ce que corrobore les valeurs des coefficients de variation de la durée du gavage $(0,16)$ et, dans une moindre mesure, du poids du foie $(0,25)$.

Les héritabilités que nous estimons représentent uniquement la variabilité génétique additive de la voie maternelle des mulards gavés. En absence d'épistasie et d'interaction de la femelle avec son conjoint, elles sont donc comparables à la moitié de ce que nous obtiendrions en souche pure. Deux raisons ont conduit à ce choix. En premier lieu, la connaissance des paramètres génétiques de la population des mulards est de peu d'intérêt pour mieux définir les caractères à améliorer chez la cane lorsque l'on choisit, comme c'est le cas ici, de pratiquer une sélection en souche pure et non une sélection pour améliorer le croisement. De plus, dans notre cas, les valeurs des paramètres différeraient d'une année à une autre car les souches de mâles de Barbarie sont différentes. En second lieu, le mulard est un hybride vrai chez lequel les première et les quatrième paires de chromosomes diffèrent morphologiquement d'une espèce à l'autre (Mott et al, 1968), ce qui interdit la synapse des chromosomes homologues. Dans cette situation les covariances entre apparentés classiquement évaluées n'ont plus de justification biologique, nous sommes alors sans méthode pour estimer les paramètres génétiques de la population des mulards.

L'effet du père est traité comme un effet fixé en raison de leur faible nombre et de leur échantillonnage non aléatoire. Dans la mesure où le père et la mère n'interagissent pas, cette correction est licite et n'introduit pas de biais dans l'estimation des paramètres génétiques de la population des mères. Une interaction existe entre les souches paternelles et maternelles du mulard (Mialon, 1985). On peut en inférer l'existence d'une interaction au niveau des reproducteurs eux-mêmes. La correction de l'influence du père absorbe alors une part de la variabilité des mères. Il est donc probable que les estimées obtenues soient biaisées. Introduire le mâle comme un effet aléatoire n'aurait rien changé à ce problème car il n'est pas plus aisé de tenir compte d'une aptitude spécifique au croisement.

Aucun auteur cité aux tableaux III, IV et V n'estime les paramètres à l'aide d'un modèle prenant en compte la totalité des parentés; ils utilisent le plus souvent un modèle père mère et calculent l'héritabilité à partir de la composante père; ils estiment donc, comme nous, la variabilité génétique additive. La méthode 3 d'Henderson (1953) est le plus souvent retenue, ce qui permet de tenir compte dans un seul modèle d'influences autres que celles des ascendants. Dans cette 
Tableau IV. Héritabilités estimées en race Barbarie sur des mâles gavés.

\begin{tabular}{lll}
\hline Caractère & Valeur & Auteur \\
\hline Poids $53 \mathrm{j}$ & 0,42 & Farran $(1980)$ \\
Poids $63 \mathrm{j}$ & 0,33 & Babilé $(1989)$ \\
Poids $67 \mathrm{j}$ & 0,43 & Farran $(1980)$ \\
Poids $81 \mathrm{j}$ & 0,17 & Farran $(1980)$ \\
Poids $84 \mathrm{j}$ & 0,41 & Babilé $(1989)$ \\
Poids $84 \mathrm{j}$ & 0,55 & Fernandez (1988) \\
Poids plumé & 0,31 & Babilé $(1989)$ \\
Poids éviscéré & 0,12 & Farran $(1980)$ \\
Paletot & 0,37 & Babilé $(1989)$ \\
Paletot & 0,63 & Fernandez $(1988)$ \\
Paletot & 0,33 & Farran $(1980)$ \\
Foie & 0,20 & Babilé $(1989)$ \\
Foie & 0,40 & Fernandez (1988) \\
Foie & 0,25 & Farran $(1980)$ \\
Magret & 0,27 & Babilé $(1989)$ \\
Magret & 0,43 & Fernandez (1988) \\
Poids cuisse & 0,33 & Babilé (1989) \\
Exsudat lipidique & 0,31 & Farran $(1980)$ \\
Exsudat lipidique & 0,59 & Fernandez (1988) \\
Exsudat lipidique & 0,23 & Babilé (1989) \\
\hline
\end{tabular}

Tableau V. Corrélations génétiques estimées chez le canard mâle de Barbarie gavé.

\begin{tabular}{llll}
\hline Caractère 1 & Caractère 2 & Valeur & Auteurs \\
\hline Poids $63 \mathrm{j}$ & Poids $84 \mathrm{j}$ & 0,88 & Babilé $(1989)$ \\
Poids $84 \mathrm{j}$ & Paletot & 0,88 & Babilé (1989) \\
Poids $84 \mathrm{j}$ & Paletot & 0,72 & Fernandez (1988) \\
Poids $84 \mathrm{j}$ & Foie & 0,38 & Babilé $(1989)$ \\
Poids $84 \mathrm{j}$ & Foie & 0,28 & Fernandez (1988) \\
Poids $84 \mathrm{j}$ & Magret & 0,56 & Babilé (1989) \\
Poids $84 \mathrm{j}$ & Magret & 0,52 & Fernandez (1988) \\
Poids $84 \mathrm{j}$ & Exsudat & 0,31 & Babilé (1989) \\
Poids $84 \mathrm{j}$ & Exsudat & 0,22 & Fernandez (1988) \\
Poids $84 \mathrm{j}$ & Cuisse & 0,76 & Babilé $(1989)$ \\
Paletot & Foie & $-0,63$ & Farran $(1980)$ \\
Paletot & Foie & 0,55 & Fernandez (1988) \\
Paletot & Foie & 0,13 & Babilé $(1989)$ \\
Paletot & Magret & 0,59 & Fernandez (1988) \\
Paletot & Exsudat & 0,43 & Fernandez (1988) \\
Foie & Magret & 0,43 & Babilé (1989) \\
Foie & Magret & 0,21 & Fernandez (1988) \\
Foie & Exsudat & 0,93 & Fernandez (1988) \\
Magret & Exsudat & 0,16 & Fernandez (1988) \\
Magret & Cuisse & 0,75 & Babilé (1989) \\
Cuisse & Foie & $-0,02$ & Babilé (1989) \\
\hline
\end{tabular}


méthode les résultats sont biaisés lorsque les paramètres sont estimés dans une souche sélectionnée; c'est le cas des résultats de Kosba et al (1981), Farran (1980), Fernandez (1988) et Babilé (1989). En revanche, dans un modèle tel que le nôtre où toutes les relations de parentés des femelles sont considérées et à condition de remonter les généalogies jusqu'aux fondateurs, ce qui est notre cas, la sélection ne biaise pas les estimations (Gianola et al, 1989; Im et al, 1989).

Des valeurs d'héritabilités en race Pékin sur des animaux non gavés figurent au tableau III et en Barbarie sur des animaux gavés, au tableau IV. Dans tous les cas, les valeurs citées des héritabilités des caractères identiques à ceux que nous analysons sont supérieures à celles que nous obtenons; ceci est normal puisque nous estimons environ la moitié des valeurs intrapopulation. Mais à une exception prés, l'héritabilité du poids du magret obtenu par Fernandez (1988) $(0,43)$, les différences sont faibles. Chez le lapin, les estimations d'héritabilités obtenues avec les mêmes données, d'une méthode qui prend en compte l'ensemble des parentés, sont supérieures à celles d'une méthode qui ne tient compte que de parentés partielles (Baselga et al, 1982; Garcia, 1981). C'est probablement pour cela que nos estimations sont supérieures à la moitié de celles obtenues en souche pure.

Les valeurs des corrélations génétiques que nous estimons entre le poids à la mise en gavage, le poids du paletot, le poids du magret et de ses composantes sont voisines de celles rapportées dans la littérature (tableau V). En revanche nous trouvons une opposition génétique entre le poids de foie ou de l'exsudat lipidique et les autres caractères; seul Farran (1980) mentionne un antagonisme d'intensité plus forte que le nôtre. Les autres auteurs trouvent des corrélations de valeurs plutôt faibles mais positives. Les différences importantes de valeurs des corrélations entre le poids du paletot et le poids du foie sont imputables à des erreurs d'échantillonnage. Si nos résultats reflètent vraiment la réalité, il existerait, génétiquement parlant, un optimum de maturité et de développement musculaire du caneton lors de sa mise en gavage garant d'un foie lourd de qualité.

\section{CONCLUSION}

À notre connaissance, c'est la première fois que, chez le canard, on utilise un modèle mixte prenant en compte la totalité des parentés. C'est également la première fois que les paramètres génétiques d'une souche de canes communes sont estimés en utilisant les performances de leurs fils hybrides gavés. Le mulard est l'animal d'intérêt économique dans le processus d'amélioration du canard commun pour la production de magret et de foie gras; en utilisant ses performances pour estimer les paramètres génétiques de sa souche maternelle, nous appréhendons mieux la réalité économique et zootechnique. C'est une manière aussi d'attribuer une valeur pour des caractères mesurables uniquement en gavage à un animal, tel la cane Aliénor, inapte à produire du foie gras lorsqu'on le gave.

Nous nous intéressons ici uniquement à la voie femelle; des progrès aussi importants peuvent être obtenus par la voie du mâle Barbarie père des mulards. Nous pourrions estimer les paramètres génétiques de la souche de Barbarie en une démarche analogue à celle que nous venons de réaliser. 


\section{REMERCIEMENTS}

Nous remercions pour leur participation à la réalisation expérimentale les techniciens animaliers de la Sica-Sepalm; nous remercions l'ITAVI pour le suivi en élevage et en gavage; nous remercions aussi l'ensemble des éleveurs et des gaveurs qui ont généré les données analysées.

\section{RÉFÉRENCES}

Babilé R (1989) Lạ production de foies gras de canards de Barbarie (Caïrina moschata) : aspects génétiques, nutritionnels et technologiques. Thèse, Institut polytechnique de Toulouse

Babilé R, Matheron G, Poujardieu B (1988) La production de foie gras de canards mâles de Barbarie. Relation entre la croissance et la production de foie. Ann Zootech 37, 219-252

Baselga M, Blasco A, Garcia F (1982) Parametros genéticos de caracteres economicos en poblaciones de conejos. In : 2do Congr Mundial de Genetica aplicada a la Production Ganadera, Madrid, Espagne, 4-8 octubre 1992, Editorial Garsi, Madrid 28, vol 6, 471480

Brun JM, Guy G, Poujardieu B, Rousselot-Pailley D, Rouvier R (1995) Composition anatomique de canards mulards de 4 génotypes : influence de l'âge et du gavage. Ann Zootech 44 (sous presse)

Clayton GA, Powell JC (1979) Growth, food conversion, carcass yields and their heritabilities in ducks (Anas platyrhyncos). Br Poult Sci 20, 121-127

Farran MT (1980) Contribution à l'amélioration de la production de foie gras à partir de canards de Barbarie mâles. Thèse, Institut polytechnique de Toulouse

Fernandez X (1988) Étude de la variabilité génétique d'une population de canards de Barbarie mâles sur les caractères de croissance et de gavage de ses descendants. Mémoire de fin d'étude, École nationale supérieure agronomique de Toulouse

Garcia F (1981) Genética y seleccion de caracteres reproductivos en el conejo de carne. Thèse, Université Polytechnique, Valence, Espagne

Gianola D, Fernando RL, Im S, Foulley JL (1989) Likelihood estimation of quantitative genetic parameters when selection occurs : models and problems. Genome 31, 768-777

Groeneveld E. Kovac M (1990a) A generalized computing procedure for setting up and solving mixed linear models. J Dairy Sci 73, 513-531

Groeneveld E, Kovac M (1990b) A note on multiple solutions in multivariate restricted maximum likelihood covariance component estimation. J Dairy Sci 73, 2221-2229

Guy G, Rousselot-Pailley D, Gourichon D (1993) Comparaison des performances quantitatives et qualititatives du gavage de l'oie, du canard mulard et du canard de Barbarie. $1^{\text {res }}$ Journées de la recherche sur les palmipèdes à foie gras, Bordeaux, France, 29 avril 1993, ITAVI Paris, 3-11

Henderson CR (1953) Estimation of variance and covariance components. Biometrics 9, 226-252

Im S, Fernando RL, Gianola D (1989) Likelihood inferences in animal breeding under selection : a missing-data theory viewpoint. Genet Sel Evol 21, 399-414

Kosba MA, Negm AM, El-Sayed TM (1981) Heritability, phenotypic and genetic correlations between breast mean weight and carcass traits in ducks. Alex $J$ Agric Res 29, 453-464

Mialon MM (1985) Croisement entre 3 souches de Barbarie et de canes communes : performances de reproduction, de gavage des mulards obtenus. Mémoire de fin d'étude. École nationale des ingénieurs des travaux agricoles (Bordeaux) 
Mott CL, Lillian H, Lockhart LH, Rigdow RH (1968) Chromosomes of the sterile hybrid duck. Cytogenetics 7, 403-412

Pingel H (1990) Genetics of growth and meat production in waterfowl. In : Poultry breeding and genetics. (RD Crawford, ed), Elsevier, New York, 913-933

Roussely M, Guichard F, Sazy E (1993) Performances zootechniques et qualité des produits du canard mulard : analyse de la variabilité individuelle. $1^{\text {res }}$ Journées de la recherche sur les palmipèdes à foie gras, Bordeaux, France, 28 avril 1993, ITAVI Paris, 87-105

Sazy E, Héraut F, Robin N (1993) Évolution de la composition corporelle des canards mulards pendant le gavage. $1^{\text {res }}$ Journées de la recherche sur les palmipèdes à foie gras. Bordeaux, France, 29 avril 1993, ITAVI Paris, 47-61 